\title{
Coevolution of dispersal in a parasitoid-host system
}

\author{
Darren Michael Green ${ }^{\mathrm{a}}$
}

July 23, 2009

a Institute of Aquaculture, University of Stirling, Stirling, Stirlingshire FK9 4LA, UK. E-mail address: darren.green@stir.ac.uk (D.M. Green).

\section{Abstract}

Interspecific interactions and the evolution of dispersal are both of interest when considering the potential impact of habitat fragmentation on community ecology, but the interaction between these processes is not well studied. We address this by considering the coevolution of dispersal strategies in a host-parasitoid system. An individual-based host-parasitoid metapopulation model was constructed for a patchy environment, allowing for evolution in dispersal rates of both species. Highly rarefied environments with few suitable patches selected against dispersal in both species, as did relatively static environments. Provided that parasitoids persist, all parameter values studied led to stable equilibria in dispersal rates for both species. There was a tendency towards higher dispersal rates in parasitoids due to the asymmetric relationships of the two species to the patches: vacant patches are most valuable for hosts, but unsuitable for parasitoids, which require an established host population to reproduce. High host dispersal rate was favoured by high host population growth rate, and in the parasitoid by high growth rates in both species.

Keywords: Competition $\bullet$ Difference equation • Dispersion $\bullet$ Nicholson-Bailey model 


\section{Introduction}

In patchy landscapes, dispersal is potentially as important as reproductive life-history traits in determining overall fitness (Clobert et al. 2001), and the optimal dispersal strategy for a species will depend upon the nature of this environment (Bowler and Benton 2005). The hierarchical nature of population dynamics in such metapopulations is well studied (Hanski 1999), as is the evolutionary ecology of the dispersal process itself (Gandon and Rousset 1999; Heino and Hanski 2001; Murrell et al. 2002). Metapopulations provide a stabilising effect on populations where local population fluctuations are to a degree independent. Thus, local extinction does not necessarily imply extinction of the whole population. Too low a dispersal rate, and colonisation cannot keep pace with the rate of local extinction on small patches. However, at very high dispersal rates, population dynamics across the whole landscape become synchronised, and the stabilising effect of the metapopulations is lost (Reeve 1990).

Species do not exist in isolation. Various studies have considered dispersal in interacting species, both in the field (Rauch and Weisser 2007; see Elzinga et al. 2007 for a summary) and theoretically (Reeve 1990; Rohani and Ruxton 1999; Briggs and Hoopes 2004; Lett et al. 2005). However, the evolutionary ecology of dispersal in interacting species is not well studied. What are the evolutionary dynamics of dispersal strategies in interacting species, and how does the strategy adapted by one species depend upon its competitor, prey, or predator species? Here, we address this with a theoretical study, using an individual-based model of the evolution and coevolution of dispersal traits in a host-parasitoid system.

Both dispersal and coevolutionary interactions of hosts and parasitoids have long been the focus of studies of evolutionary biology, both theoretically (Hochberg 1997; Fellowes and Travis 2000; Godfray and Sasaki 2000; Sasaki 2000; Bonsall et al. 2002) and experimentally (Fellowes and Godfray 2000; Green et al. 2000; Weisser 2000; Bonsall et al. 2002). Their interactions are closer than that found in many other forms of symbiosis, especially the potentially tight coevolutionary interactions of highly host-specific koinobiont parasitoids (Janzen 1980; Askew and Shaw 1986), which allow the host - which they develop on or within - to continue development after parasitism. We here consider a model appropriate for a system such as the fruit fly Drosophila melanogaster and its Hymenopteran parasitoids, including Asobara tabida, Pachycrepoideus vindemiae or Leptopilina boulardi: short-lived, tightly interacting species reliant on ephemeral, patchy, resources. Intraspecific competition is an important driver in the evolution of dispersal (Bowler and Benton 2005). The traditional model of host-parasitoid interaction, the Nicholson-Bailey (N-B) model (Nicholson and Bailey 1935), implicitly accounts for intraspecific competition in the parasitoid. Here, we amend the model to account for intraspecific competition in the host in a complementary manner as has been done by other authors (e.g., Beddington et al. 1975).

\section{Model}

\section{Single-patch model}

In brief, there are three key features of our two-species metapopulation model: First, within-patch dynamics are modelled using the N-B model, formulated to account for intraspecific host competition. Second, the landscape consists of multiple patches, divided into those suitable and those unsuitable for host reproduction. Patches switch between these states according to a simple Markov process. Third, both hosts 
and parasitoids disperse across patches, with the propensity to disperse being an inherited trait subject to mutation.

The classical Nicholson-Bailey (1935) model of host-parasitoid dynamics is as follows:

$$
\begin{gathered}
H_{t+1}=R H_{t} F_{t} \\
P_{t+1}=H_{t}\left(1-F_{t}\right) \\
F_{t}=\exp \left(-a P_{t}\right)
\end{gathered}
$$

Here, $H$ denotes the number of of hosts, $P$ the number of parasitoids, and $F$ the proportion of hosts that escape parasitism and go on to reproduce. In the absence of parasitoids, the host population grows with growth factor $R$; and $a$ denotes the searching efficiency of the parasitoids.

This model can be derived from some simple assumptions concerning the behaviour of the hosts and parasitoids, but is unsatisfactory in that the maximum size of host and parasitoid populations are unbounded. The model developed in this paper steps back to an individual-based approach which constrains the populations of hosts and parasitoids to a maximum size dependent on host carrying capacity. It is assumed that larval hosts show scramble competition, as has been demonstrated for Drosophila melanogaster and D. simulans (Miller 1964). Scramble competition is implemented as suggested by Beddington et al. (1975) and by Brännström and Sumpter (2005), which follows the Ricker model (Ricker 1954). In contrast, intraspecific competition amongst parasitoids is modelled assuming contest competition. The main model concepts are illustrated in Fig. S1 in Electronic Supplementary Material.

At time $t$, the population consists of sets $H_{t}$ of adult hosts and $P_{t}$ of adult parasitoids, which both reproduce asexually. The model 'patch' consists of $n$ sites, each of which provides enough resources to produce one host or parasitoid. Each host makes a number of visits drawn from a Poisson distribution with mean $R$, amongst the $n$ sites (at random, with replacement), depositing one egg per visit. Each parasitoid similarly makes a number of visits drawn from a Poisson distribution with mean $A=a n$ (thus $A$ and $R$ have the same units), depositing one egg at each visited sites with host eggs. What emerges from each site $k$ at the end of timestep $t$ depends upon the form of the competition, and the numbers of host eggs $\left(\eta_{k}\right)$ and parasitoid eggs $\left(\pi_{k}\right)$ laid. Under scramble competition, no adult stage of either species is produced if $\eta_{k} \neq 1$. If $\eta_{k}=1$, then an adult host is produced if $\pi_{k}=0$ or an adult parasitoid otherwise (contest competition).

As with the classical N-B model, all individuals survive for one time-step only. A deterministic representation of this model, equivalent to that given by Beddington et al. (1975) is given by the following set of equations, as shown in Fig. S2 in ESM. The correspondence between these two modelling approaches is explained in Appendix A.

$$
\begin{gathered}
H_{t+1}=F_{t} R H_{t} \exp \left(\frac{-R H_{t}}{n}\right) \\
P_{t+1}=\left(1-F_{t}\right) R H_{t} \exp \left(\frac{-R H_{t}}{n}\right) \\
F_{t}=\exp \left(\frac{-A P_{t}}{n}\right)
\end{gathered}
$$

The basic N-B model has no stable states for $H>0$ and $P>0$. In particular, it shows oscillations of ever-increasing magnitude in both $H$ and $P$ for many combinations of $a$ and $R$. The presence of a carrying capacity for the host population in the model presented here is a fundamental difference, substantially altering the population dynamics. The dynamics of the single-species Ricker model (Eq(s). 2; obtained where $A=0$ ) are complex, showing a range of behaviour from a single stable state, with period doubling through limit cycles to chaos as $R$ is varied, as shown in Fig. S3 in ESM for a single patch. Including a second species increases the complexity of the model (Fig. 
S3). The concern of this paper lies, however, in examining the model at the much longer-term level of the evolutionary and coevolutionary dynamics.

\section{Multiple-patch model}

The single-patch model described above is extended to multiple patches with migration between them. The model 'world' consists of $N$ patches, each of which is divided into the $n$ sites, $N n$ sites in total. It is assumed that inter-patch distance is small compared with possible lifetime travel distance, as may be the case for various parasitoid species (Elzinga et al. 2007), and thus local spatial dynamics are less important. Accordingly, a spatially implicit metapopulation model is developed where all patches are modelled as effectively equidistant from each other. The state of each patch $i(1 \leq i \leq N)$ at time $t$ is denoted by $S_{i, t}$. Each patch can be 'active' $\left(S_{i, t}=1\right)$, in which case it provides resources to support host larval development, or 'inactive' $\left(S_{i, t}=0\right)$, in which it does not. Patches switch between states according to a first-order Markov process, with inactive patches at time $t$ becoming active at time $t+1$ with probability $\lambda$, and active patches similarly becoming inactive with probability $\mu$. Patches thus persist according to a form of geometric distribution, remain for mean duration $\frac{1}{\mu}$ time steps, and the proportion of patches active is given by $\frac{\lambda}{\lambda+\mu}$.

At the beginning of each time step $t$, the population consists of a set of newly emerged adult hosts and parasitoids each identified by an index $j$, located at patch $s_{j}$ each with an inherited dispersal trait $\rho_{j}$. The population of hosts and parasitoids on patch $i$ are denoted by sets $H_{i, t}$ and $P_{i, t}$. Individual $j$ disperses with probability $\rho_{j}$, in which case it is relocated to a patch $s_{j}^{\prime}$ at time $t^{\prime}$ (indicating the time after dispersal for time-step $t$ ), drawn at random from the whole population of patches, whether active or inactive: $s_{j}^{\prime}=\operatorname{Uniform}(1, N)$; otherwise, $s_{j}^{\prime}=s_{j} . H_{i, t}^{\prime}$ and $P_{i, t}^{\prime}$ then represent the sets of hosts and parasitoids at this new time $t^{\prime}$ in patch $i$ for which $s_{j}^{\prime}=i$.

For all active patches $S_{i, t}=1$, local reproduction and parasitism proceeds according to the individual-based simulation above. For inactive patches, $H_{i, t+1}$ and $P_{i, t+1}=\emptyset$. The trait value of an individual emerging from a site is derived from that of its parent, subject to mutation. Mutation was modelled in two ways. First, it occurs with probability $m$, in which case $\rho_{j}$ is assigned a random number in the range $[0,1]$, regardless of the parent trait. This allows long-distance changes in trait value, as might be appropriate where a trait is encoded by few loci. Alternatively, for a more polygenic trait, mutation was modelled using cross-generational increments in $\rho$ chosen from a normal distribution with mean zero and standard deviation $\sigma$, subject to the bounds $0 \leq \rho_{j} \leq 1$. Where $\sigma=\frac{1}{3} \sqrt{\frac{\pi}{2}} m$ the mean difference in $\rho$ between generations is equal in the two methods (Appendix B). Preliminary results showed no qualitative differences in equilibrium behaviour between the two models and so the simpler $m$-based one is used throughout below.

\section{Simulation}

The model has seven parameters, listed in Table 1. Throughout, we use a mutation rate $m=0.001$, sufficient to allow evolution without qualitatively affecting any equilibria, and a fixed number of patches $N=100$. A model run was initialised with 10000 hosts and 1000 parasitoids, distributed randomly across the $N$ patches, each with a value of $\rho$ chosen independently from the uniform distribution $(0,1)$. The starting population did not affect the long-term evolutionary dynamics. Each simulation was run for 500 time units, which preliminary analysis showed to be sufficient to allow coevolutionary dynamics to reach a quasi-equilibrium, subject to stochastic 
fluctuation (below, simply "equilibrium").

Means of total host population size $\bar{H}$, parasitoid population size, $\bar{P}$, host

dispersal rate $\overline{\rho_{H}}$ and parasitoid dispersal rate $\overline{\rho_{P}}$ were recorded and averaged across the last 100 time units of the simulation. Population dynamics in the absence of dispersal were studied with single-patch simulations of size $n=10000$. The evolutionary dynamics with only a single species evolving were studied with simulations in which the other species had a fixed, constant dispersal trait for all individuals.

\section{Results}

\section{Population dynamics}

At small patch sizes, stochastic extinction of the parasitoids occurred on average at the 39th time-step for $n=250$, and at the 15th for $n=200$ under the parameters used in Fig. 1. For higher patch sizes, the evolutionary responses $\rho$ varied little with patch size as shown in Fig. 1, though this is not the case for the population dynamics. Therefore to simplify the analysis of dispersal rate, $n$ is fixed at 500 throughout below - a level sufficiently high that stochastic extinction is a relatively unimportant factor for most parameter combinations examined.

Mean size of host and parasitoid populations are shown as a function of $\rho_{H}$ and $\rho_{P}$ (fixed dispersal strategies) in Fig. S5 in ESM. At the highest and lowest parasitoid dispersal rates, host population size is negatively correlated with parasitoid population size, as these parasitoid dispersal strategies are less successful, resulting in lower parasitism pressure reducing the size of the host population. For the lowest host dispersal rates, host population size is in turn low, with a concomitant reduction in parasitoid population size. Otherwise, for higher $\rho_{H}$ and intermediate $\rho_{P}$, population sizes are less sensitive to the values of $\rho$.

\section{Evolutionary dynamics}

The evolutionary response of dispersal strategy of each species to different fixed strategies in the opposing species is shown in Fig. 2 for three different combinations of patch-dynamic parameters $\lambda$ and $\mu$, plus permanently active patches. Where the lines are not parallel to the axes, the optimum dispersal trait for one species depends upon the dispersal strategy adopted by the opposing species. This suggests possibilities for coevolutionary dynamics. That the pairs of lines for the two species intersect suggests an equilibrium is possible for both dispersal traits in a full coevolutionary model, but does not in itself dictate whether such an equilibrium would be stable or unstable. Therefore, simulations allowing for coevolution of both species were performed.

Simulations allowing both species to coevolve confirmed the presence of a single stable equilibrium in dispersal rate for both species, located at the point in trait space predicted by the single-species evolution models (Fig. 2). Where these equilibria for $\bar{\rho}$ are intermediate between 0 and 1 , the populations of both parasitoids and hosts show peaked distributions of $\rho$ around these means (Fig. 3). No branching of populations into low- and high-dispersal-strategy sub-populations was observed for either species using either model of mutation. Parasitoid dispersal rate $\overline{\rho_{P}}$ was found to be consistently lower than that for the host population (Fig. 4). This is reflected in the mean within-patch host-parasitoid dynamics, demonstrated in Fig. 5 as a function of patch age.

\section{Dispersal with varied patch dynamics}

Equilibrium dispersal rates for both species depend upon both patch-dynamic and life-history parameters, as shown in Fig. 2 and more fully explored in Fig. 4. 
Lower $\lambda / \mu$ decreases the density of active patches, and at a sufficiently low patch density, populations do not persist. Where patches are permanently active, equilibrium dispersal rate for both species is high, especially so for hosts, and the optimal value of dispersal in one species depends only weakly on the dispersal trait adapted by the other (Fig. 2). However, even in the absence of inactive patches, dispersal traits do not reach fixation with every individual dispersing. Without patch dynamics, patches are more homogeneous, removing a selection pressure for increased dispersal. Higher dispersal traits are found where the majority of patches are active, though with a fast turnover of active patches. For hosts, the main determiner of dispersal rate is the patch activation rate $\lambda$ (Fig. 4). Higher $\lambda$ increases active patch density with no effect on active patch lifetime, benefitting dispersal.

The same effect applies to parasitoid dispersal with higher dispersal rate at higher $\lambda$. However, parasitoid dispersal rate is additionally affected by patch removal rate $\mu$. Higher $\mu$ results in a shorter patch lifetime, giving a greater benefit to dispersal. This effect may be pronounced only for parasitoids, since whereas host density is highest in new active patches, density of parasitoids is highest for older patches (Fig. 5).

\section{Dispersal with varied life-history parameters}

For the lowest levels of the parasitoid searching parameter $A$, parasitoid populations do not persist; however, too high a value for $A$ and in a stochastic model, the host population is driven to extinction. Similarly, $R$ is constrained by being high enough to support a host population with extreme values of $R$ causing frequent stochastic extinction through the Ricker process. Where either the parasitoid or both species do not persist, blank areas are seen in the lower panels of Fig. 4 (the host population in the absence of parasitoids not being modelled here).

Host dispersal is selected for under conditions of high $R$ (Fig. 4). Here, intense competition between hosts and high host fitness in empty, active patches benefits increased dispersal. Host dispersal rate is largely independent of $A$, with some reduction in $\overline{\rho_{H}}$ for low $A$. However, parasitoid dispersal rate is highly dependent upon $R$. Parasitoid dispersal is selected for where both $R$ and $A$ are high. Four selection pressures contribute to this result: First, host dispersal and reproduction rates are also high, and newly active patches will establish host populations quickly. Second, parasitoid population density is high and intra-patch competition amongst parasitoids strong. Third, high $A$ increases the relative benefit of dispersing to newly established host populations to avoid intraspecific competition. Fourth, with very high $R$, local host extinction becomes more likely.

\section{Discussion}

Dispersal is a double-edged sword: it promises benefits of locating unoccupied habitat and reduced competition, but carries risk of lower fitness or mortality if such habitat is not found. The model formulation above implicitly assumes that parasitoid and host competitive ability is unaffected by $\rho_{j}$, and that neither hosts nor parasitoids can determine the value of a new patch before settling upon it. The implicit cost of dispersing is therefore the risk that the patch travelled to is of poorer quality than that left behind, with the potential benefit that a new, unoccupied (for hosts) or unparasitised (for parasitoids) patch may be discovered. Other authors have included terms for explicit costs of dispersal (e.g., Murrell et al. 2002). Here, no such energy cost is included, but dispersal is automatically costly in a naturalistic manner under our 
model framework, where individuals disperse to an inactive patch, with higher cost in more rarefied environments. Other potential costs could include Allee effects where reproduction is more difficult in sparsely populated patches.

These benefits and costs are asymmetrical, due to the different relationships of hosts and parasitoids to the patches. Host reproduction is quickest in newly active, vacant patches free from intraspecific competition. Parasitoids, however, require an established host population before they can reproduce. Therefore, there is a greater probability of dispersing to an unsuitable patch for a parasitoid than for a host. With a lower dispersal rate in the parasitoid and a need for an established host population before parasitoid reproduction, there is a lag between host colonisation of a newly active patch and the establishment of a resident parasitoid population (Fig. 5). Once established, the parasitoid population reduces host density in subsequent generations. This lower dispersal in the parasitoid is in agreement with field studies, with several showing relatively restricted levels or distances of dispersal in parasitoids (Elzinga et al. 2007). Mostly short-distance dispersal, with sufficient long-distance dispersal to account for long-distance colonisation (Elzinga et al. 2007) could result from a dispersal kernel with a heavy tail, as might be generated through power-law Lévy flights (Viswanathan et al. 1999). The fruit fly $D$. melanogaster shows fractal periods of activity which might generate such flight patterns (Cole 1995).

Our model assumes that larval competitive ability is unaffected by parasitism, which would be most appropriate where parasitism occurs after larval competition, as is the case for Drosophila pupal parasitoids such as Pachycrepoideus vindemiae rather than larval parasitoids such as Leptopilina spp. Also, both host and parasitoid dispersal occur before reproduction and after intraspecific competition, which is also reasonable for Drosophila and its parasitoids. However, this will not be the same for all species interactions. Dispersal is selectively advantageous where kin competition is avoided by dispersing individuals (Hamilton and May 1977; Bach et al. 2006; Poethke et al. 2007), as is the case where dispersal occurs earlier than larval intraspecific competition. For systems where the dispersing host stage is itself parasitised, selection on parasitoid dispersal rate may still operate via the potential of parasites to manipulate the dispersal behaviour of their hosts (Thomas et al. 2005; Lion et al. 2006), potentially leading to direct coevolutionary arms-races. For example, the optimal parasitoid dispersal rate may depend upon the number of other species it can parasitise, and the rate at which its subpopulations are subject to stochastic extinction even where hosts persist.

Dispersal as modelled above is not plastic. Whether dispersal is advantageous over a whole lifetime, and at a specific instant can be treated as separate questions. For example the presence of a parasitoid can be a proximal trigger for dispersal, as seen in the production of winged aphid morphs (Slogett and Weisser 2002). More plastic dispersal strategies, for example where dispersal rates are density dependent (Travis et al. 1999), could be a future addition to our model. Models show that plastic dispersal strategies are selectively advantageous and will evolve where there is the potential to do so (Travis et al. 1999).

The model predicts that change in the dispersal trait of one population can drive reciprocal change in the dispersal trait of the other species. This is not a direct selection pressure, but operates via the population dynamics - the 'ecological loop' of Bowler and Benton (2005):

Changing dispersal rates in one species alters the population dynamics of both, leading to changes in fitness, driving the 
evolution of dispersal in the second species.

As habitats become further fragmented through habitat destruction, species are increasingly reliant on dispersal between patches to maintain viable populations. However, dispersal is not necessarily in the interest of the individual where dispersal is costly (Travis et al. 1999). Habitat fragmentation causes greater susceptibility of species to extinction and lower species diversity, especially at higher trophic levels (Bascompte and Solé 1998; Gilbert et al. 1998; Bonsall et al. 2002; Elzinga et al. 2007), with extinction of one species leading to further extinction of other, linked, species (Memmott et al. 2006). Given that the optimal dispersal strategy for a species will depend on the nature of its environment (Bowler and Benton 2005), habitat change and destruction may lead to selection pressures acting on dispersal strategies. Use of pest management strategies such as introduction of parasitoids of pest species may similarly shift the optimum for pest dispersal traits. Where polymorphism in dispersal trait exists (e.g., Appleby and Credland 2001), selection may favour different morphs, potentially compromising the efficiency of pest control.

\section{Acknowledgements}

With thanks to Charles Godfray and Istvan Kiss for helpful comments on the manuscript.

\section{Appendix A}

Assume a single patch of size $n$ sites with populations $H_{t}$ of hosts and $P_{t}$ of adult parasitoids. Each host makes a Poisson distributed number of independent egg-laying 'visits' to sites at random with mean $R$. Similarly, each parasitoid makes a Poisson-distributed number of visits with mean $A$. Therefore, the number of visits made to individual sites are in turn Poisson distributed as $\eta \sim \operatorname{Poisson}\left(R H_{t} / n\right)$ for hosts and $\pi \sim \operatorname{Poisson}\left(A P_{t} / n\right)$ for parasitoids. Given this distribution, the probabilities of a site being visited once by hosts, and zero times by parasitoids are given by:

$$
\begin{gathered}
p(\eta=1)=\frac{R H_{t}}{n} \exp \left(-\frac{R H_{t}}{n}\right) \\
p(\pi=0)=\exp \left(-\frac{A P_{t}}{n}\right) .
\end{gathered}
$$

Visits are independent for hosts and parasitoids, therefore multiplying gives the probabilities of a site having one host visit and no parasitoid visits $p(\pi=0) p(\eta=1)$, which sites give rise to a host; and the probability of one host visit and $>0$ parasitoid visits $(1-p(\pi=0)) p(\eta=1)$, which sites give rise to a parasitoid. Multiplying by the number of sites $n$ and rearranging gives Eqns. (2) above. Alternatively, where $n=1, p(\pi=0)$ is equivalent to the original equation for $F$ in Eqns. (1).

\section{Appendix B}

The mean absolute difference between two random numbers chosen independently and uniformly in the range $[0,1]$ is given by $\frac{1}{3}$. The mean absolute value of numbers chosen from the standard normal distribution is given by $\sqrt{\frac{2}{\pi}}$. As a first-order rule to allow comparison between the two mutation models (assuming random drift of $\rho$ ), we therefore apply $\frac{1}{3} m=\sqrt{\frac{2}{\pi}} \sigma$, and thus $\sigma=\frac{1}{3} \sqrt{\frac{\pi}{2}} m$. 


\section{References}

Appleby JH, Credland PF (2001)

Bionomics and polymorphism in

Callsobruchus subinnotatus

(Coleoptera: Bruchidae). Bull

Entomol Res 91:235 - 244

Askew RR Shaw MR (1986) Parasitoid communities: their size, structure and development. In: Waage JK, Greathead D (eds) Insect parasitoids. London, Academic Press, pp 225 264

Bach LA, Thomsen R, Pertoldi C, Loeschcke, V (2006) Kin competition and the evolution of dispersal in an individual-based model. Ecol Model 192:658 - 666

Bascompte J, Solé RV (1998) Effects of habitat destruction in a prey-predator metapopulation model. J Theor Biol 195:272 - 282

Beddington JR, Free CA, Lawton JH (1975) Dynamic complexity in predator-prey models framed in difference equations. Nature 255:58 $-60$

Bonsall MB, French DR, Hassell MP (2002) Metapopulation structures affect persistence of predator-prey interactions. J Anim Ecol 71:1075 1084

Bowler DE, Benton TG (2005) Causes and consequences of animal dispersal strategies: relating individual behaviour to spatial dynamics. Biol Rev 80:205 - 225

Brännström Å, Sumpter DJT (2005) The role of competition and clustering in population dynamics. Proc R Soc B 272:2065 - 2072 doi:10.1098/rspb.2005.3185

Briggs CJ, Hoopes MF (2004) Stabilizing effects in spatial parasitoid-host and predator-prey models: a review. Theor Popul Biol 65:299 - 315
Clobert J, Nichols JD, Danchin E, Dhondt A (2001) Dispersal. Oxford University Press, Oxford, UK

Cole BJ (1995) Fractal time in animal behaviour: the movement activity of Drosophila. Anim Behav 50:1317 1324

Elzinga JA, Nouhuys S van, Leeuwen D-J van, Biere A (2007) Distribution and colonisation ability of three parasitoids and their herbivorous host in a fragmented landscape. Basic Appl Ecol 8:75 - 88

Fellowes MDE, Godfray HCJ (2000) The evolutionary ecology of resistance to parasitoids by Drosophila. Heredity $84: 1-8$

Fellowes MDE, Travis JMJ (2000) Linking the coevolutionary and population dynamics of hostparasitoid interactions. Popul Ecol 42:195 - 203

Gandon S, Rousset F (1999) Evolution of stepping stone dispersal rates. Proc R Soc B 266:2507 - 2513

Gilbert A, Gonzalez A, Evans-Freke I (1998) Corridors maintain species richness in the fragmented landscapes of a microecosystem. Proc R Soc B 265:577 - 582

Godfray HCJ, Sasaki A (1999) A model for the coevolution of resistance and virulence in coupled host-parasitoid interactions. Proc R Soc B 266:455 463

Green DM, Kraaijeveld AR, Godfray HCJ (2000) Evolutionary interactions between Drosophila melanogaster and its parasitoid Asobara tabida. Heredity 85:450 458

Hamilton WD, May RM (1977) Dispersal in stable habitats. Nature 269:578 581 
Hanski I (1999) Metapopulation ecology. Oxford University Press, Oxford UK

Heino M, Hanski I (2001) Evolution of migration rate in a spatially realistic metapopulation model. Am Nat 157:495 - 511

Hochberg ME (1997) Hide or fight? The competitive evolution of concealment and encapsulation in parasitoid-host associations. Oikos 80:342 - 352

Janzen DH (1980) When is coevolution? Evolution 34:611 - 612

Lett C, Auger P, Fleury F (2005) Effects of asymmetric dispersal and environmental gradients on the stability of host-parasitoid systems. Oikos 109:603 - 613

\section{Lion S, van Baalen M, Wilson WG} (2006) The evolution of parasite manipulation of host dispersal. Proc R Soc B 273:1063 - 1071

Memmott J, Alonso D, Berlow E, Dobson A, Dunne J, Solé R, Weitz J (2006) Biodiversity loss and ecological network structure. In: Pascual M, Dunne AJ (eds) Ecological Networks: Linking Structure to Dynamics in Food Webs. Oxford University Press, Oxford UK, pp $325-347$

Miller RS (1964) Larval competition in Drosophila melanogaster and $D$. simulans. Ecology 45:132 - 148

Murrell DJ, Travis JMJ, Dytham C (2002) The evolution of dispersal distance in spatially-structured populations. Oikos 97:229 - 236

Nicholson AJ, Bailey VA (1935) The balance of animal populations. Part 1. Proc Zool Soc Lond 3:551 - 598

Poethke HJ, Pfenning B, Hovestadt T (2007) The relative contribution of individual and kin selection to the evolution of density-dependent dispersal rates. Evol Ecol Res 9:41 50

Rauch G, Weisser WW (2007) Local and spatial dynamics of a host-parasitoid system in a field experiment. Basic Appl Ecol 8:89 - 95

Reeve JD (1990) Stability, variability, and persistence in host-parasitoid systems. Ecology 71:422 - 426

Ricker WE (1954) Stock and recruitment. J Fisheries Res Board Can 11:559-623

Rohani P, Ruxton GD (1999) Dispersal-induced instabilities in host-parasitoid metapopulations. Theor Popul Biol 55:23 - 36

Sasaki A (2000) Hostparasite coevolution in a multilocus gene-for-gene system. Proc R Soc B $267: 2183-2188$

\section{Sloggett JJ, Weisser WW (2002)}

Parasitoids induce production of the dispersal morph of the pea aphid, Acyrthosiphon pisum. Oikos 98:323 333

Thomas F, Adamo S, Moore J (2005) Parasitic manipulation: where are we and where should we go? Behav Process 68:185 - 199

Travis JMJ, Murrell DJ, Dytham C (1999) The evolution of density-dependent dispersal. Proc R Soc B 266:1837 - 1842

Viswanathan GM, Buldyrev SV, Havlin S, da Luz MGE, Raposo EP, Stanley HE (1999) Optimizing the success of random searches. Nature 401:911 914

Weisser WW (2000) Metapopulation dynamics in an aphid-parasitoid system. Entomol Exp Appl 97:83 - 92 
Table 1. Ranges of parameter values used (favoured values given in parentheses).

\begin{tabular}{llc}
\hline$N$ & Number of patches & 100 \\
$n$ & Patch area (carrying capacity) & $250-1600(500)$ \\
$\lambda$ & Rate of patch activation & $0.0367-0.333(1 / 4)$ \\
$\mu$ & Rate of patch inactivation & $0.0367-0.333(1 / 4)$ \\
$R$ & Growth factor of hosts without parasitoids & $2-18(8)$ \\
$a$ & Patch area searched by a single parasitoid & $0.008-0.072 \times n(0.016)$ \\
$A$ & & $4-36(8)$ \\
$m$ & Mutation rate & 0.001 \\
\hline
\end{tabular}




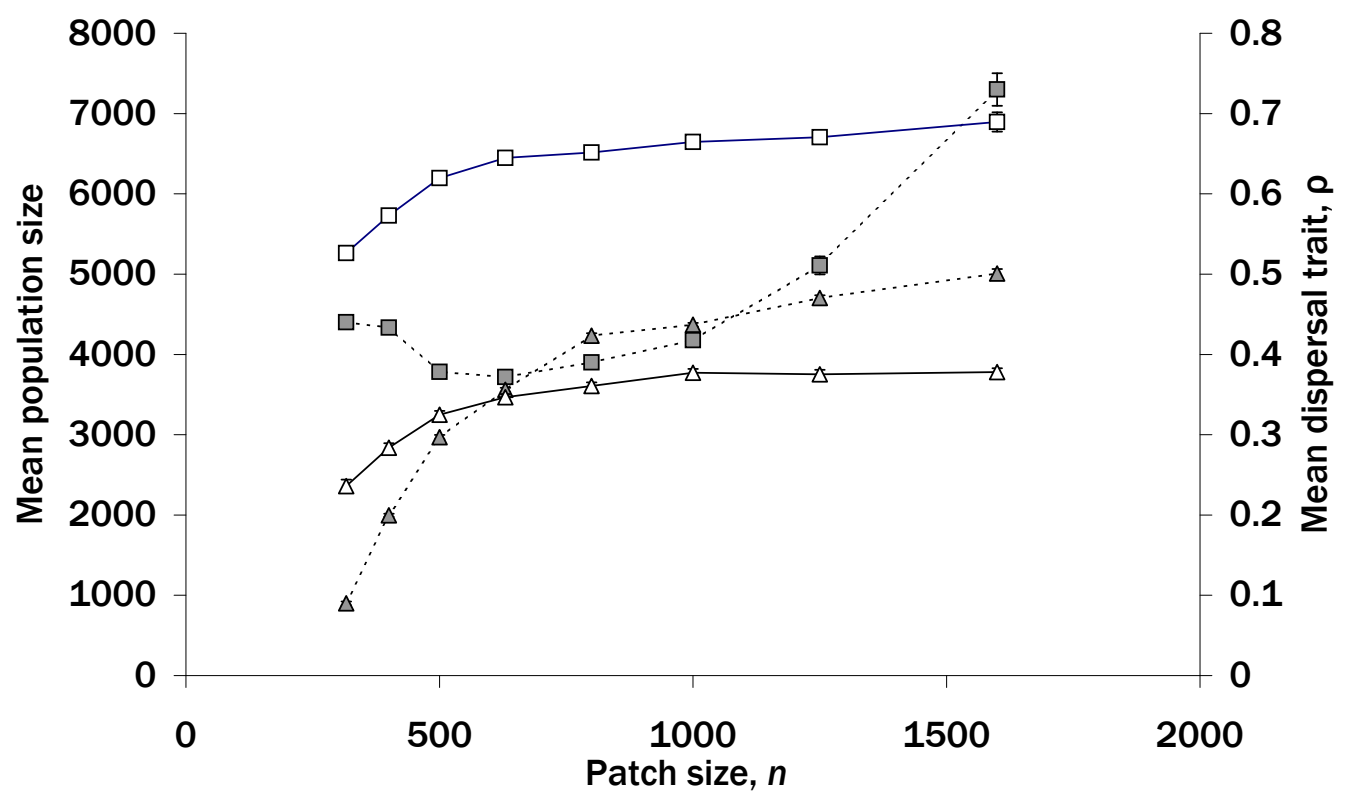

Figure 1. Equilibrium conditions versus patch size $n$. Numbers of hosts $(\bar{H}, \mathbf{\square})$ and parasitoids $(\bar{P}, \mathbf{\Lambda})$ are shown by dotted lines and the left-hand axis; mean dispersal rate for hosts $\left(\overline{\rho_{H}}, \square\right)$ and parasitoids $\left(\overline{\rho_{P}}, \triangle\right)$ are shown by solid lines and the right-hand axis. Default parameters (Table 1). Standard errors are included for both variables (each of 16 replicates), but generally too small to be shown. 


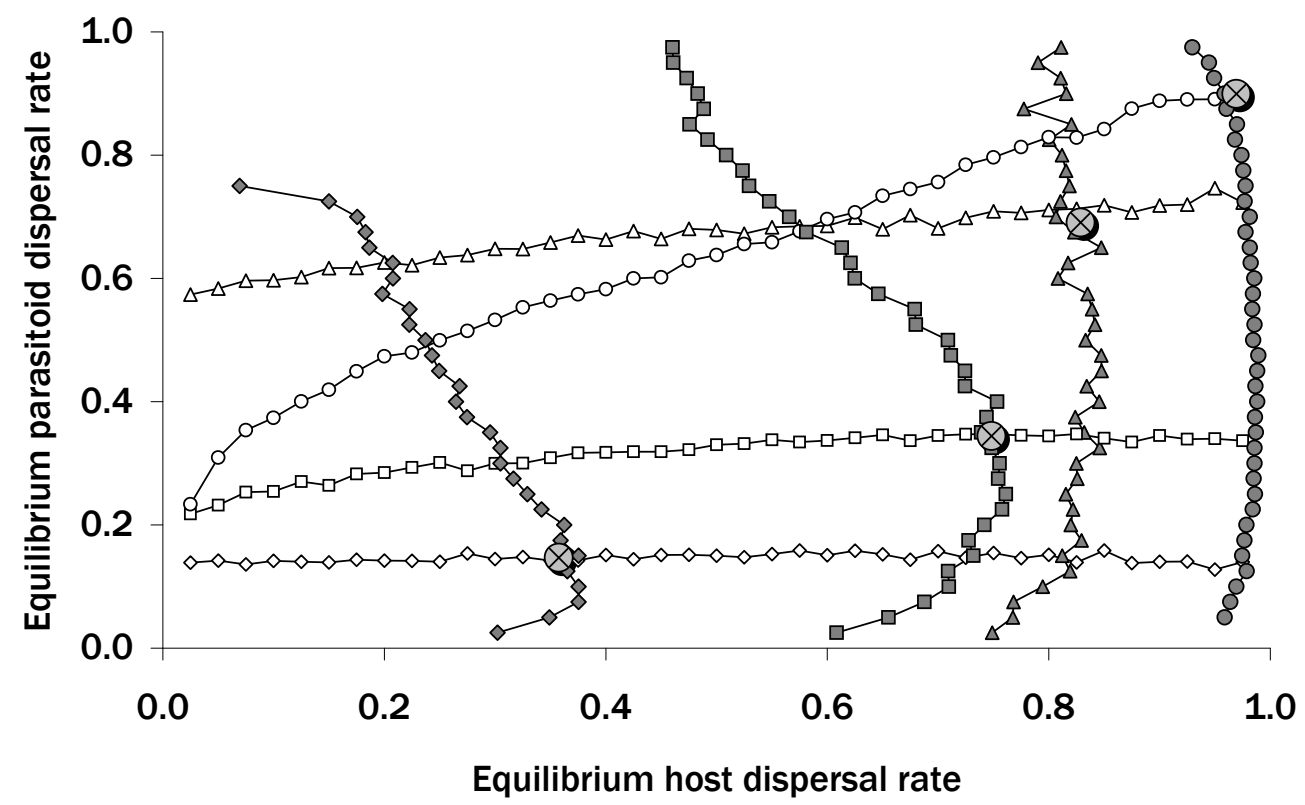

Figure 2. Equilibrium conditions with evolution of one or two of the interacting species. Open symbols: equilibrium parasitoid $\overline{\rho_{P}}\left(y\right.$-axis) with fixed host $\rho_{H}$ ( $x$-axis). Filled symbols: equilibrium host $\overline{\rho_{H}}\left(x\right.$-axis) for fixed parasitoid $\rho_{P}$ ( $y$-axis). Mean durations of patch activity $\left(\mu^{-1}\right)$ and inactivity $\left(\lambda^{-1}\right)$ were $\triangle$ : always active; $\square: 4$ active, 4 inactive; $\circ: 3$ active, 1.5 inactive; $\diamond: 10$ active, 20 inactive. Default life-history parameters (Table 1). Means of ten simulations are shown for each point. Larger crossed circles at the intersections indicate where both species were allowed to evolve (means of 30 simulations). Some extreme dispersal values led to extinction. 


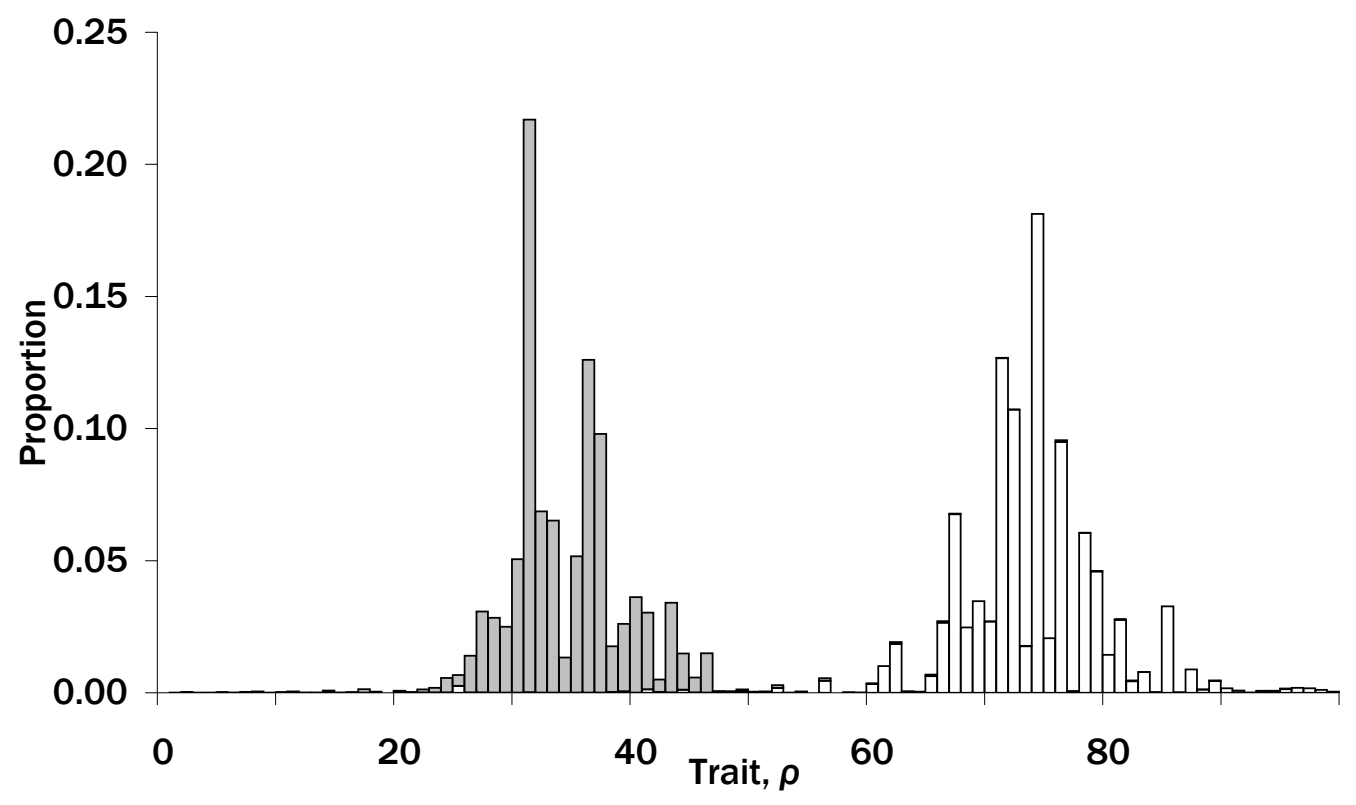

Figure 3. Distribution of final host $\rho_{H}$ (open symbols) and parasitoid $\rho_{P}$ (filled symbols), aggregated over 10 model runs. Default parameters (Table 1). 

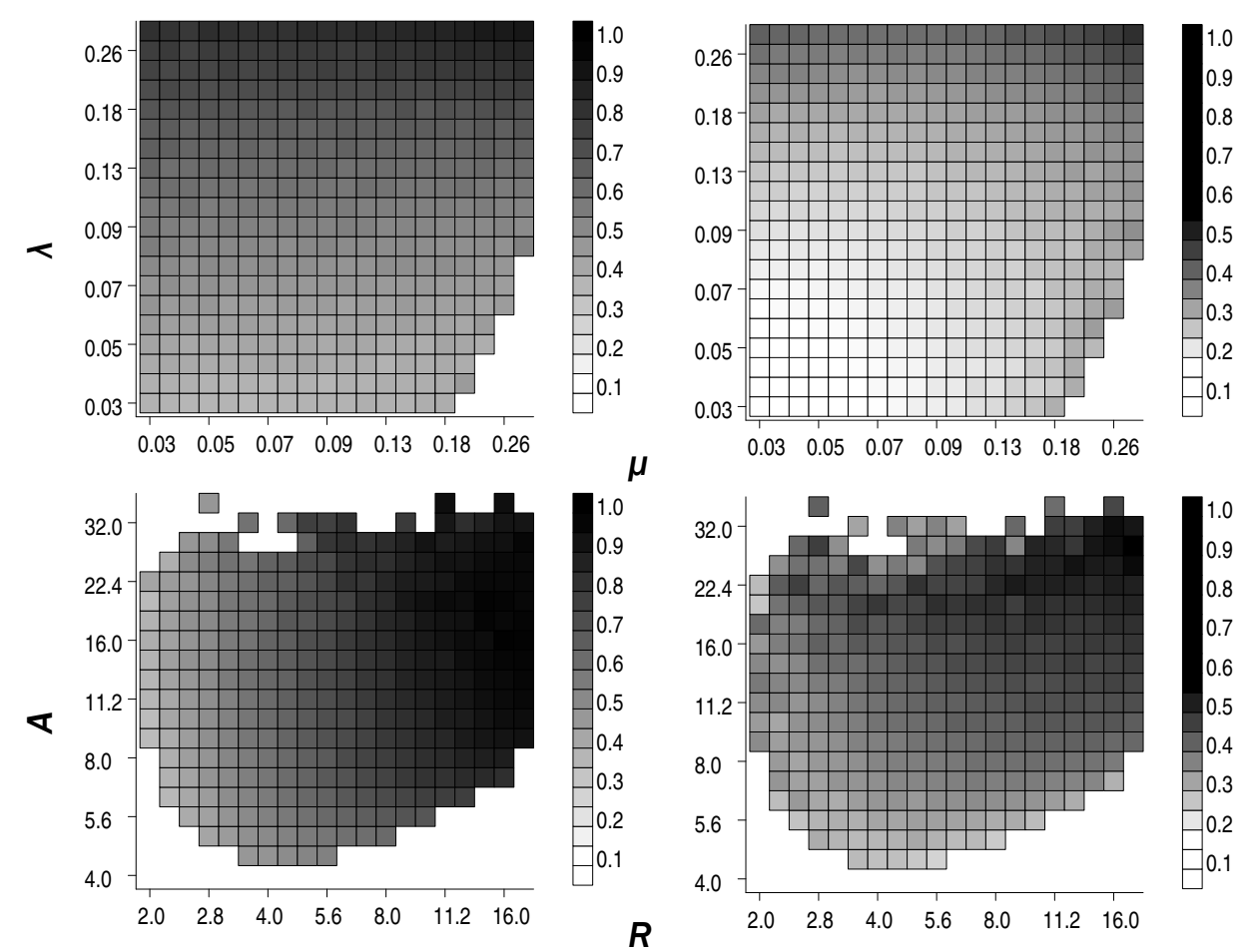

Figure 4. Long-term means for $\overline{\rho_{H}}$ (left) and $\overline{\rho_{P}}$ (right) versus varied patch dynamic parameters (top) and host and parasitoid parameters (bottom). Top: $\mu$ ( $x$-axis) versus $\lambda$ (y-axis); bottom: $R_{0}$ ( $x$-axis) versus $A$ (y-axis). Means of 15 simulations are plotted (or fewer with stochastic extinction). Regions where one or both species do not persist are shown empty. A colour version is provided in ESM (Figure S4). 


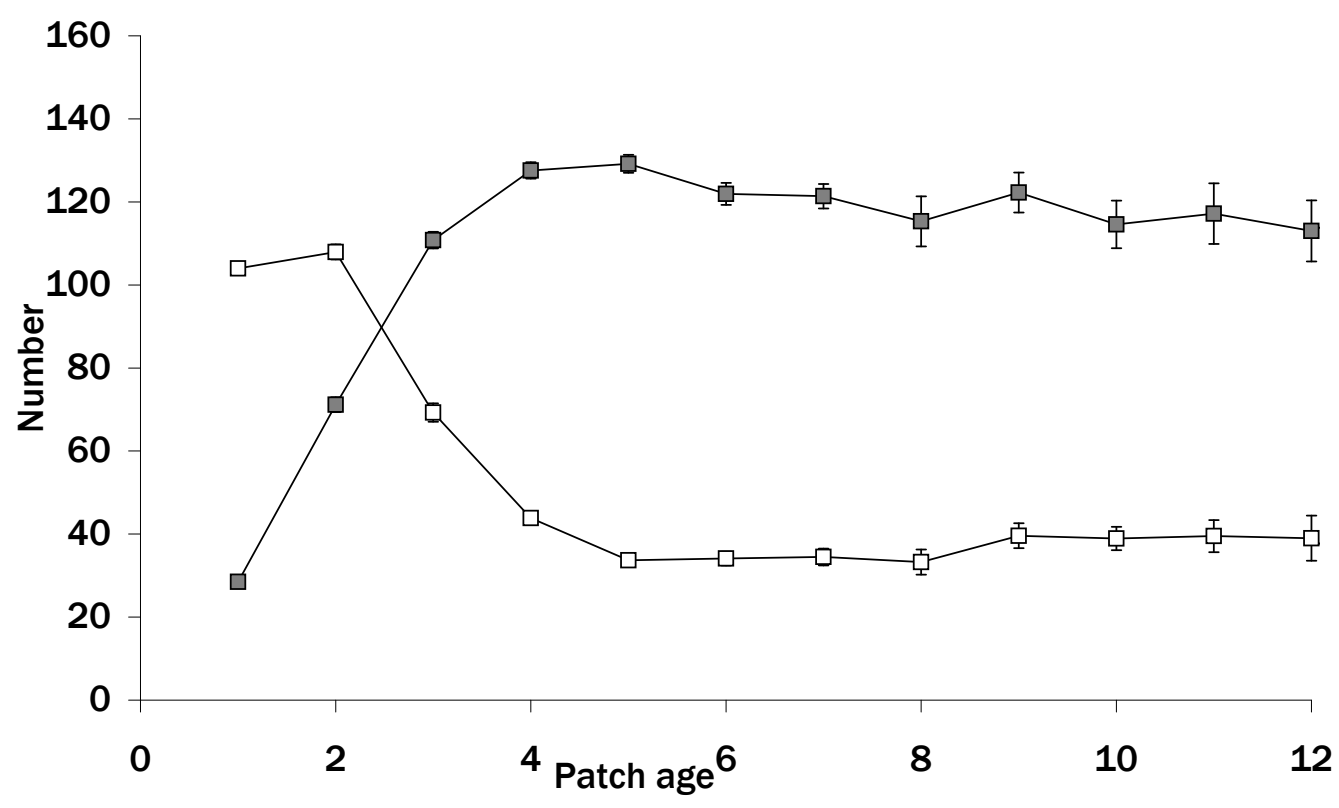

Figure 5. Mean numbers of hosts (open symbols) and parasitoids (filled symbols) in a given patch versus patch age (time steps), accumulated over 10 model runs. Default parameters (Table 1). Standard errors are included, but generally too small to be shown. 